\title{
Achsenzivilisationen und die Genese der Moderne im Lichte einer Theorie langfristigen sozialen Wandels
}

\section{Heinz-Jürgen Niedenzu}

Zusammenfassung: In der gegenwärtigen Soziologie hat die Entwicklung einer sachhaltigen Theorie langfristigen sozialen Wandels keinen besonderen Stellenwert. Als Folge dieses Mankos bleibt die Genese der Moderne als einer neuartigen soziokulturellen Organisationsform sowie der ablaufenden Globalisierungsprozesse weitgehend unverstanden, solange die Erklärungen nur eine geschichtlich, räumlich und kulturell limitierte Perspektive aufweisen. Am Beispiel der These von Eisenstadt zur Bedeutung der sog. Achsenzivilisationen für ein Verständnis der Moderne soll aufgezeigt werden, dass seine Wandlungstheorie einer Einbettung in eine umfassendere Theorie soziokultureller Evolution bedarf. Machtprozesse und im wechselseitigen Verbund mit diesen die Entwicklung historisch gebundener Weltinterpretationen weisen den Weg für ein vertieftes Verständnis der strukturellen Bedeutung der Achsenzivilisationen für das Aufkommen der Moderne.

Schlüsselwörter: Evolutionstheorie, langfristiger sozialer Wandel, Gesellschaftsformationen, Weltbilder, Achsenzivilisationen, Moderne, multiple modernities

\section{Axial Civilizations and the Emergence of Modernity from the Perspective of a Theory of Long-Term Social Change}

\begin{abstract}
In recent sociology the modelling of long-term social change doesn't play any important role in the great contemporary theoretical conceptions. As a result of this the emergence of modernity as a
\end{abstract}

H.-J. Niedenzu $\bowtie$

Universität Innsbruck, Institut für Soziologie

Universitätsstraße 15, 6020 Innsbruck, Österreich

E-Mail: Heinz-Juergen.Niedenzu@uibk.ac.at 
completely new form of sociocultural organization and the ongoing processes of globalization remain to a great extent opaque. Explications are most often socio-historically, regionally and culturally restricted in perspective. At the example of the analysis of axial civilizations of Shmuel N. Eisenstadt and their meaning for the understanding of modernity I will make clear that his theory of social change only takes on greater significance within a larger theory of sociocultural evolution. Processes of power together with the development of historically bounded conceptions of the world lead the way for a more elaborated understanding of the structural impact of the axial civilizations for the formation of modernity.

Keywords: Evolutionary theory, macro-social processes of change, development of social systems, conceptions of the world, axial civilizations, modernity, multiple modernities

Es ist wohl keine Übertreibung zu behaupten, dass die Entwicklung oder zumindest die Weiterarbeit an vorliegenden Entwürfen einer Theorie langfristigen sozialen Wandels im Sinne einer Theorie sozialer/soziokultureller Evolution, die auf die theoriegeleitete Rekonstruktion der Geschichte menschlicher Gesellschaftsformen seit ihren Anfängen abzielt, in der rezenten Soziologie auf kein besonderes Interesse stößt. So fußen gegenwärtige Analysen etwa zum Aufkommen und zur Form der Moderne oder zu Globalisierungsprozessen in aller Regel auf einer historischen Tiefenperspektive, die, bezogen auf das verarbeitete geschichtliche Material, wenn überhaupt selten mehr als die letzten Jahrhunderte umfasst. Hinzu tritt häufig noch ein regionaler und kultureller Bias in der Analyse. Eine Einfügung dieser Analysen in eine umfassendere Wandlungstheorie bleibt ein Desiderat. Dies gilt sowohl für die klassischen Modernisierungstheorien als auch für die Dependenztheorien, aber auch für die explizit historisch-prozessual argumentierende Weltsystemanalyse, und selbst postkoloniale Analysen als ideologiekritische Gegenposition zu den traditional westzentrischen Ansätzen und Denkmustern sind nicht frei von derartigen perspektivischen Beschränkungen.

Diese Abstinenz überrascht, war doch die Entstehungsphase der Soziologie oder allgemeiner der Sozialwissenschaften speziell im 19. Jahrhundert sehr stark mit makrohistorischen Entwicklungsfragen befasst (Niedenzu 2014; 2016). Dieses Denken findet sich in den zum Teil stark evolutionistisch-teleologisch konstruierten Entwicklungs- und Stadienmodellen von Comte, Spencer und natürlich auch Marx wieder, bis hin zu einer abgeschwächten Version bei Durkheim. Ohne hier ins Detail gehen zu können lässt sich festhalten, dass ein in einem weiten Sinne verstandenes evolutionär orientiertes Denken in der Frühphase mithin eine Selbstverständlichkeit war, welches aber in der Folgezeit weitestgehend aus dem Interessenfokus geriet. Dementsprechend konstatierte Jürgen Habermas (1982b, S. 129) anlässlich der Eröffnung des 17. Deutschen Soziologentags 1974 zu Recht das Fehlen einer Theorie zur Erklärung der sozialen Evolution, ja noch nicht einmal eine angemessene Konzeptualisierung läge vor. Gleich- 
zeitig pointiert die Feststellung von Habermas aber auch eine soziologiegeschichtliche Wende hin $\mathrm{zu}$ einer gewissen, wenn auch nicht nachhaltigen Renaissance des Interesses an Fragen langfristiger Entwicklungen in der zweiten Hälfte des 20. Jahrhunderts. In der Soziologie wie auch in der Kultur- und Sozialanthropologie wurden klassische Fragestellungen wieder virulenter, einerseits verbunden mit einer theoretischen Absatzbewegung vom damals dominanten Strukturfunktionalismus, andererseits mittels einer Wiederaufnahme von Marxschen Fragestellungen zu makrohistorischen Prozessen. In der deutschsprachigen Soziologie wäre hier das von Habermas (1982) und Eder (1973; 1976) ausgearbeitete Evolutionsmodell und die in diesem Kontext stattgefundene Debatte zu nennen, des Weiteren aber auch das von Dux (1982; 2000) verfolgte Programm der Entwicklung einer allgemeinen Theorie Sozialen Wandels. Auch in der Luhmannschen Systemtheorie finden sich evolutionstheoretische Anleihen, wobei er jedoch keine stringente und materialgestützte Entwicklungstheorie ausgearbeitet hat (Niedenzu 2012, S. 94 ff.). In der angelsächsischen Soziologie wurde die Evolutionsthematik von Parsons (1977) zumindest gestreift, blieb aber in ihrer Bedeutung für sein Theoriemodell marginal. Debattiert wurden und werden jedoch Entwicklungs- und Stadienmodelle, die ursprünglich in der kulturund sozialanthropologischen Literatur präsentiert wurden und in der Soziologie auf Resonanz stießen, etwa bei Sanderson (1990 und 2001; Sanderson and Alderson 2005) oder bei W.G. Runciman (1989; 2009), die um eine soziologisch-theoretische Fundierung makrohistorischklassifizierender und stadial angelegter Modelle des Wandels von gesellschaftlichen Organisationsformen bemüht sind. Generell kann also von einer, wenn auch disziplinär gesehen marginal verbleibenden, vorübergehenden Renaissance neoevolutionären Denkens gesprochen werden (Meleghy und Niedenzu 2003).

Evolution, soziale Evolution, soziokulturelle Evolution, Menschheitsgeschichte, Geschichte - so unterschiedlich diese Begriffe bezüglich ihres Erklärungsanspruchs und die zu erfassenden Prozesse auch sein mögen, so fußen sie doch auf zwei Erkenntnisdimensionen, die im neuzeitlichen Wissensbestand unabdingbar miteinander verbunden sind und unsere Weltsicht leiten: grundlegend geht es um ein Verständnis von Entwicklung als dem zeitlichen Nacheinander von sozialen Geschehnissen und gesellschaftlichen Strukturformen, die nicht mehr periodisch-zyklisch und/oder strukturell weitgehend unverändert, sondern linear-fortschreitend in veränderter oder auch gänzlich neuer Form, somit zukunftsoffen, aufeinander folgen. Mit diesem neuen Zeitverständnis geht ein Denken und Reflexionsvermögen einher, welches sich der dynamischen Prozessualität natürlicher Abläufe als auch der menschlichen Eingriffs- und Gestaltungsmacht insbesondere auch mit Bezug auf gesellschaftliche Verhältnisse zunehmend bewusst wird. Unabhängig von außermenschlichen Mächten und Kräften wird damit die Möglichkeit des kontinuierlichen Wandels von gegebenen soziokulturellen Strukturformen im Sinne ihrer Entwicklungsfähigkeit, aber auch die Emergenz völlig neuer Strukturformen, die mit der Kontinuität von Entwicklungen brechen, als realistisch und eigenmächtig umsetzbar denkbar. Das Aufkommen dieser Vorstellungen von Zeit und damit auch von Evolution, Entwicklung und 
Wandel hat einen spezifischen historisch-gesellschaftlichen Ort, ist gebunden an kognitive und soziokulturelle Bedingungslagen und daran anschließende Wissensstrukturen und Weltbilder (Elias 1989; Dux 1989).

Grundsätzlich beanspruchen die eingangs genannten Oberbegriffe, wenn auch mit unterschiedlichem Abstraktionsgrad, materiell und immateriell vorliegende Artefakte und Phänomene in einem logisch-kausal angelegten System unter den Prämissen von Zeitlichkeit und Wandlungsfähigkeit von Formen und Strukturen zeitlinear ordnen und einer wissenschaftlichen Erklärung zuführen zu können. Unter dem am weitesteten angelegten Begriff der (allgemeinen) Evolution wird dabei auf die generelle Entwicklung der Formen des Lebens fokussiert, wobei es unter dem Aspekt der zeitlichen Abfolge rein empirisch gesehen einen Trend zu ausdifferenzierteren und komplexeren organismischen Strukturen gibt, womit bei höheren Lebensformen eine zunehmende ,Emanzipation vom Diktat der Umwelt' (Reichholf 1994) einhergeht. In einem engeren Verständnis meint der Begriff der sozialen Evolution den Hominisationsprozess, also die evolutive Phase des Aufkommens humansozialer Lebensformen aus vorwegliegenden sozietären Lebensformen, während in einem weiter gefassten Verständnis darunter auch die daran anschließende Entwicklung unterschiedlichster soziokultureller Organisationsformen subsumiert wird. Der Begriff der Menschheitsgeschichte bzw. der soziokulturellen Evolution weist je nach Verwendung eine gewisse Ambivalenz auf; so sucht etwa Norbert Elias (1983) in seinem Modell der Großen Evolution nach einem entwicklungsdurchgängigen Formalprinzip, welches er in der immer komplexer werdenden Verschachtelung von Integrationseinheiten ausmacht, was unmittelbar zu seinem Figurationsverständnis führt, welches er dann auf gesellschaftliche Prozesse anwendet. Wenn demgegenüber Günter Dux (2000) davon spricht, ,die ganze Menschheitsgeschichte in den Blick zu nehmen', geht es ihm um die Rekonstruktion der Abfolge und des kausallogischen Zusammenhangs in der Entwicklung soziokultureller Organisationsformen und Wissensformen, die ihren heuristischen und empirischen Ausgangspunkt in einer humanspezifischen anthropologischen Verfassung finden. Bei beiden Autoren, und ebenso bei Habermas, impliziert der Begriff der Menschheitsgeschichte natürlich nicht die Annahme eines substanzartig verstandenen Gattungssubjekts als Träger einer kontinuierlich verlaufenden Gattungsgeschichte. Am wenigsten ambitioniert ist schließlich der in den Geschichtswissenschaften verwendete Geschichtsbegriff, der die längste Zeit sehr stark an empirisch gehaltvollen Rekonstruktionen konkreter historischer Ereignisse und Ereignisabfolgen in Form von Narrativen und weniger an das Konkrete übersteigenden abstrakten Entwicklungsmodellen gesellschaftlicher Organisationsformen orientiert war.

Im Folgenden möchte ich in einem ersten Schritt, ausgehend von einer älteren Replik von Habermas auf Luhmann, das begriffliche und konzeptionelle Verhältnis von Geschichte (Geschichtswissenschaft) und Evolution (soziologische Entwicklungstheorie) neu aufgreifen. Habermas kam damals zu dem Schluss, dass die Soziologie über keine elaborierte Evolutionstheorie verfügt. Warum aber bedarf es überhaupt einer evolutiv informierten Tiefenperspektive 
für die Analyse rezenter gesellschaftlicher Formationen? In einem zweiten Schritt soll diesbezüglich untersucht werden, inwieweit die mit geschichtlichem Material überaus gesättigte Eisenstadtsche Untersuchung der sog. Achsenzivilisationen als tiefenhistorischem Ausgangspunkt für seine These der multiple modernities in der Lage ist, die eingangs genannten Kurzschlüssigkeiten bisheriger entwicklungstheoretischer Angebote zu überwinden. Abschließend soll die Fruchtbarkeit seiner Wandlungsthese, die exemplarisch ja eine Teilphase innerhalb der soziokulturellen Evolution thematisiert, anhand offener Fragen bezüglich ihrer Bedeutung für die Entwicklung einer allgemeinen Theorie langfristigen sozialen Wandels diskutiert werden. Mein erster Kritikpunkt betrifft dabei die Notwendigkeit einer klareren evolutionstheoretischen Einbettung und Positionierung, der zweite die mangelnde Berücksichtigung der Entwicklung menschlicher Kognition. Beide Punkte zusammengenommen erlauben uns, so meine These, einerseits sowohl der historischen Tiefendimension der Strukturdimension der Achsenzivilisationen inklusive ihrer kontingenten Ausprägungen als auch der gestiegenen Reflexivität auf die Verhältnisse als einem Kernmerkmal dieser Epoche und ihre langfristige Bedeutung für die Analyse der Moderne theorietechnisch besser Rechnung tragen zu können. Andererseits liefern sie wichtige Hinweise für die Erfordernisse, die an eine Theorie langfristigen sozialen Wandels bzw. der soziokulturellen Evolution zu stellen sind.

\section{Geschichte und Evolution}

Das Verhältnis zwischen Geschichtswissenschaft auf der einen und Soziologie auf der anderen Seite lässt sich nur schwer eindimensional auflösen, gibt es doch weder die Geschichtswissenschaft noch die Soziologie. Auf der Seite der ersteren hat Habermas (1982a) mit der Unterscheidung von Geschichtsforschung und Geschichtsschreibung gearbeitet. Grundsätzlich bedienen sich ihm zufolge historische Darstellungen einer Erzählform. Die Geschichtsforschung strukturiert dabei die Narration der Ereignisse und Ereignisketten, mithin die eigentliche Geschichtsschreibung, durch theoretische und methodologische (Vor-)Entscheidungen für die Art und Weise der Aufarbeitung der empirischen Artefakte, wobei es diesbezüglich in den letzten Jahrzehnten zu einer ,Soziologisierung der Geschichtsschreibung' etwa durch die Analyse institutionsgegebener Handlungsspielräume sowie in Ansätzen zu einer globalhistorischen Ausrichtung gekommen ist. Als, diachrone Sozialforschung ' kann diese dann dazu führen, ,[...] die provinzielle Enge des Anwendungsbereichs vieler (soziologischer, meine Ergänzung) Theorien, die mit Allgemeinheitsanspruch auftreten [...]“ (Habermas 1982a, S. 202), nachzuweisen. In diesem Sinne fällt den historischen Wissenschaften, heuristisch gesehen, sowohl die Rolle eines Lieferanten empirischer Daten für als auch die Rolle eines Korrektivs von historisch ansetzenden soziologischen Theorien bis hin zu Theorien soziokultureller Evolution zu (vgl. Habermas 1982a, S. 244). Auf der Seite der Soziologie ist andererseits festzuhalten, dass sich die großen 
Paradigmen des Fachs wie auch feldspezifische Theorien einer abstrakt-ahistorischen Begriffsarchitektur bedienen, die auf universale humansoziale Vergesellschaftungsstrukturen und -prozesse abzielt. So können die Kategorien und Theorien auf die unterschiedlichsten historischen und soziokulturellen Situationen angewendet werden, wobei sich das historisch Konkrete jeweils als adaptive Form des Abstrakten manifestiert. Beispiele dafür wären auf der Mikroebene Rollen-, Sozialisations- und Identitätstheorien, auf der Meso- und Makroebene Institutionen-, Macht- und Differenzierungstheorien.

Während auf dieser Abstraktionsebene die Korrekturfunktion der Geschichtswissenschaft weitgehend wegfällt, tritt sie umso stärker in den Vordergrund, wenn es um die empirische Validität zeit- und raumbezogener soziologischer Analysen, beispielsweise um dem Wandel der Familienformen oder des Geschlechterverhältnisses in der europäischen Neuzeit, geht. Noch stärker wird die Abhängigkeit vom historischen Material, wenn es um eine soziologische Aufarbeitung und Systematisierung der Menschheitsgeschichte im Sinne einer Entwicklungstheorie und damit um das Konzept der sozialen bzw. soziokulturellen Evolution geht. Hier sieht Habermas jedoch eine unüberwindbare Kluft zwischen Geschichtswissenschaft und (s)einer Entwicklungstheorie, die ihrerseits ,[...] auf Annahmen über universale Bewußtseinsstrukturen und entwicklungslogisch angeordnete Lernniveaus [...]“ (vgl. Habermas 1982a, S. 204, 218) beruht. Diese kann ihm zufolge nicht in eine Strukturen rekonstruierende und typologisierende Universalgeschichtsschreibung, die der narrativen Form verpflichtet ist und durch diese überhaupt erst konstituiert wird, überführt werden: „Der Evolutionstheorie darf die Rolle einer Theorie der Geschichte nicht zugemutet werden, weil Geschichte als solche nicht theoriefähig ist. Die Evolutionstheorie findet ihre Anwendung nicht in Geschichtsschreibung, sondern im praktischen Diskurs" (Habermas 1982a, S. 204). Hier wird mithin eine kategoriale Differenz der jeweiligen Argumentationsgänge bezüglich langfristiger Wandlungsvorgänge postuliert. Vor diesem Hintergrund greift für Habermas (1982a, S. 230 f.) beispielsweise die Eisenstadtsche Analyse des innovativen Potentials in den Achsenkulturen zu kurz, bleibt sie doch letzten Endes, trotz aller auf die Änderung von Strukturen und von Machtverhältnissen bezogenen Untersuchungen und deren begrifflichen Systematisierung, weitestgehend narrativ. Wohl benennt Eisenstadt, wie im Übrigen auch Habermas, mit den Eliten Trägergruppen von Innovationen und damit das Aufkommen völlig neuer Lösungsvarianten auf Herausforderungen aller Art, aber die kognitiven Voraussetzungen für derartige Innovationen, also die Genese eines neuen dahinter liegenden höheren Lernniveaus und entsprechender Bewusstseinsstrukturen, bleibt in der Analyse ausgespart und sie lässt sich Habermas zufolge auch nicht narrativ abbilden:

„Wenn wir die Ebene der strukturellen Möglichkeiten (Lernniveaus) von der Ebene der faktischen Abläufe unterscheiden, lassen sich die beiden Kausalitäten mit einem Wechsel der Erklärungsperspektive erläutern. Das Eintreten eines neuen historischen Ereignisses können wir mit Bezugnahme auf kontingente Randbedingungen und auf die Herausfor- 
derung durch strukturell offenstehende Möglichkeiten erklären; hingegen erklären wir das Auftreten einer neuen Bewußtseinsstruktur mit Bezugnahme auf das entwicklungslogische Muster vorangegangener Strukturen und auf den Anstoß durch problemerzeugende Ereignisse.“ (Habermas 1982a, S. 233)

Die begriffsanalytische Ebene, auf der die soziokulturelle Evolution abgebildet werden kann, ist die von Gesellschaftsformationen, die durch hochabstrakte Regelungen, durch Organisationsprinzipien, gekennzeichnet sind (Habermas 1982b, S. 136). Diese stellen Rahmenbedingungen dar, ,[...] innerhalb welcher Strukturen Wandlungen der Institutionen- und Deutungssysteme möglich sind“" (Habermas 1982a, S. 234). Es sind im alten Rahmen nicht mehr zu lösende Problemlagen, die zu neuen Lernniveaus und damit zu neuen Organisationsprinzipien führen können, wobei der Ausgangspunkt für die Bewältigung evolutionärer Herausforderungen die Lernkapazität von Individuen ist (vgl. Preglau 2015, S. 317 f. sowie Tab. 12.1). Grundsätzlich ist aber erst der Verbund von Persönlichkeits-, Kultur- und Gesellschaftssystem evolutionsfähig, wobei die theoretische Herausforderung darin liegt, die entwicklungsrelevanten Austauschbeziehungen zwischen den Komponenten aufzuzeigen, was für Habermas bedeutet, eine entsprechende Lerntheorie zur Verfügung zu haben (Habermas 1982b, S. 133). Gleichzeitig liegt hier jedoch kein Automatismus angesichts evolutionärer Herausforderungen vor, vielmehr unterstreicht Habermas (1982a, S. 235) die relative Unwahrscheinlichkeit solcher evolutionären Schübe. Die Abfolge der Lernniveaus folgt damit wohl einerseits entwicklungslogisch nachkonstruierbaren Lernschritten, ist andererseits aber in ihrem Voranschreiten evolutionären Zufallsprozessen unterworfen. Daher lässt sie sich auch nicht in Form einer auf durchgehende Kontinuität abzielenden historischen Narration im Sinne der Geschichtsschreibung kausal begründen, wohl aber rekonstruktiv und entwicklungslogisch als solche einsichtig machen. Das bedeutet, dass die Idee eines Ganzen der Geschichte, ihrer Totalität als Menschheitsgeschichte, in evolutionärer Perspektive als heuristischer Bezugspunkt für die Rekonstruktion der Formationen und Organisationsprinzipien erforderlich ist, diese aber nicht zu verwechseln ist mit der Vorstellung eines geschichtlichen Ganzen im Sinne einer narrativ darstellbaren durchgängigen Abfolge von geschichtlichen Vorgängen:

„Vielmehr tritt das historische Material unter Bestimmungen der soziokulturellen Evolution. Diese ist kein Makroprozeß, der sich an einem Gattungssubjekt vollzieht. Träger der Evolution sind die Gesellschaft und die in ihr integrierten Handlungssubjekte zumal. Ablesen lässt sich die Evolution an einem rational nachzukonstruierenden Muster einer Hierarchie von immer umfassenderen Strukturen. Wenn wir diese Strukturen von den Vorgängen trennen, mit denen die empirischen Substrate sich verändern, brauchen wir weder Einsinnigkeit, noch Kontinuität, noch Notwendigkeit oder Irreversibilität des Geschichtsverlaufs zu unterstellen. Wir rechnen mit anthropologisch tiefsitzenden allge- 
meinen Strukturen ... Solche Grundstrukturen beschreiben den logischen Spielraum, in dem sich umfassendere Strukturbildungen vollziehen können. $O b$ es jedoch und wann es zu neuen Strukturbildungen kommt, hängt von kontingenten Umständen ab.“ (Habermas 1982a, S. 248)

Demzufolge kann es auch zu Stagnation auf einem Entwicklungsstadium oder gar zu einem entwicklungslogisch gesehen - Rückfall auf ein früheres Stadium kommen.

\section{Die evolutionstheoretische Perspektive}

Die angesprochene Kritik von Habermas an Eisenstadt ist nachvollziehbar und gerechtfertigt, wobei aber zu bedenken ist, dass das evolutionstheoretische Denken bei Eisenstadt viel stärker ans geschichtliche Material gebunden (Habermas' Einschätzung als ,narrative Analyse') denn kategorial an Gesellschaftsformationen und Organisationsprinzipien orientiert ist. Insofern verfolgt Eisenstadt eher eine empirisch ausgerichtete entwicklungstheoretische denn eine evolutionstheoretische Perspektive. Offensichtlich wird das, wenn er sich von den älteren evolutionären Stadienmodellen distanziert, weil diese in aller Regel mit gesetzmäßigen, gradlinigen und universal gültigen Entwicklungen operierten. An die Stelle der Analyse systemimmanenter Eigenschaften und Übergangsmechanismen träten, so seine Einschätzung, allgemeine Ursachen (z.B. wirtschaftliche, technologische, geistige usw.) und Trends, etwa in Richtung auf Komplexitätssteigerung. Damit setzen sie sich dem Vorwurf aus, allgemeine Trends mit den Ursachen des Wandels zu verwechseln oder die Annahme zu verfolgen, allgemeine Tendenzen könnten konkrete Wandlungen erklären (Eisenstadt 1970, S. 75). In Abhebung von diesen Ansätzen legt Eisenstadt in seiner Analyse der Achsenzivilisationen besonderen Wert darauf, die Spezifika dieser Gesellschaften und ihrer Institutionen detailliert herauszuarbeiten. Der Wandlungsmechanismus wird primär gesellschaftsendogen als ein kompliziertes Zusammenspiel von Machtstrukturen, Elitenkonstellationen und symbolischen Orientierungssystemen (Weltbilder; Religionen) konzeptualisiert. Ist der Wandlungsmechanismus somit multifaktoriell macht-, konfliktund kulturtheoretisch bestimmt, wird das Ausmaß des Wandlungspotentials der Achsenkulturen ebenfalls endogen primär aus der historisch-empirisch unterschiedlich erfolgten Auflösung der Spannung zwischen der gegebenen mundanen Ordnung und einer transzendentalen Ordnungsvorstellung, welche Basisprämissen für die Ausgestaltung der kulturellen und sozialen Ordnung zur Verfügung stellt, begründet. Im Kampf um die institutionelle Ordnung der Gesellschaft wird der Entwicklung auf der kulturellen Ebene analytisch dabei eine autonome Rolle zugestanden, auch wenn deren Trägergruppen in vielfältiger Weise mit den Trägern der politischen, ökonomischen und sozialen Machtverhältnisse verwoben sind (Eisenstadt 2009). In evolutionärer Perspektive ist dabei interessant, dass Eisenstadt das in den Achsenkulturen angelegte Wand- 
lungspotential im Sinne einer ontologischen Tiefendimension als mitursächlich für die zuerst im Westen vonstattengehende Ausbildung einer völlig neuen Organisationsform, der 'Moderne', ansieht (Arnason, Eisenstadt and Wittrock 2005).

Mit der Orientierung am von Karl Jaspers vertretenen geschichtsphilosophischen Konzept einer Achsenzeit handelt sich Eisenstadt allerdings ein Problem ein. In historischen Zeiträumen gesehen traten demnach das typisierende Merkmal der Grundspannung zwischen den Sphären des Mundanen und des Transzendentalen und die damit einhergehende gesteigerte Reflexivität auf die Verhältnisse in verschiedenen Regionen fast zeitgleich auf. Da die Eisenstadtsche Argumentation aber phänomenal ausgerichtet ist und das Wandlungspotential nur pro futuro herausgearbeitet wird, bleibt die Frage nach den Ursachen für das parallele Auftreten eines neuen Organisationsmodells (Imperien; Hochkulturen; Zivilisationen) sowie eines neuen Weltverständnisses (poly- und monotheistische Religionen) unbeantwortet. Strukturell können diese nur in den Vorbedingungen liegen, also im historischen Vorlauf, oder aber sie beruhen auf Interferenzen zwischen den Zivilisationen, die er aber weitgehend unberücksichtigt lässt. Insofern zeigt sich hier insofern ein Paradoxon, als dass er in einer werkgeschichtlich späteren Phase nunmehr einerseits grundsätzlich evolutionäre Tendenzen in der Weltgeschichte anerkennt (Eisenstadt 2009, S. 136, 143) sowie entwicklungstheoretisch bezüglich der Langzeitwirkung der Achsenkulturen argumentiert (Erste Moderne; multiple modernities), gleichzeitig aber den Ausgangspunkt seiner Argumentation punktualistisch setzt und in keinen umfassenderen prozessual-evolutionären Kontext stellt. Hier kann uns die Bezugnahme auf die allgemeine Evolutionstheorie und deren Grunderkenntnisse weiterhelfen, um das phänomenal Neue einer Erklärung zuzuführen

Evolutionstheoretisch mit Bezug auf gesellschaftliche Prozesse zu argumentieren beinhaltet, in starker Verkürzung, drei zentrale Vorstellungen (Niedenzu 2012): Evolution meint zum Einen einen kreativen Prozess, in dem Neues entstehen kann („,emergieren“), ohne dass das Neue bereits im Alten angelegt ist bzw. in Form eines Ursprungsdenken aus diesem herausgeführt oder abgeleitet werden kann. Die spätere Funktion des Neuen erklärt nicht seine Genese. Die zweite Vorstellung betrifft den Zusammenhang von Kontinuität und Bruch. Der evolutive Prozess ist insofern ein kontinuierlicher, als dass neuere Organisationsformen aus älteren entstanden sind. Das kann organisatorische Weiterentwicklung und Feinmodellierung bedeuten; es kann aber auch einen Sprung im Sinne eines qualitativen Bruchs mit völlig neuen Möglichkeiten der Gestaltung gesellschaftlicher Verhältnisse beinhalten. Beide Linien sind als parallel existierend zu betrachten, schließen sich nicht wechselseitig aus. Und schließlich kennt die Evolutionstheorie mit dem Mechanismus der Selektion und dem Kriterium des Reproduktionserfolges eine Erklärungsformel, die, adaptiert auf soziokulturelle Systeme, einsichtig machen kann, warum bestimmte gesellschaftliche Organisationsmodelle im historischen Prozess verschwunden sind, andere sich kaum verändert haben und warum neue sich dauerhaft bewährt haben. Kurzum, eine moderne soziologische Theorie der soziokulturellen Evolution beinhaltet weder geschichtsde- 
terministische Vorstellungen im Sinne von durchzulaufenden Stadien noch einen teleologisch gesetzten Endpunkt gesellschaftlicher Entwicklung. Im Prinzip lassen sich demzufolge die unterschiedlichen institutionellen Varianten, die für die Achsenzivilisationen kennzeichnend sind, auf der evolutionstheoretischen Folie von Kreativität und Emergenz, Kontinuität und Bruch mit früheren Organisationsformen sowie Umweltbewährung abbilden. Diese Lesart betrifft aber primär erst einmal die interne Varianz auf einer Entwicklungsstufe, nämlich dem Konstrukt ,Achsenzivilisation'.

In evolutionstheoretischer Perspektive muss darüberhinausgehend aber auch die vertikale Zeitachse, also die Durchgängigkeit bzw. der Bruch mit vorhergehenden Organisationsund Weltinterpretationsformen, d.h. den bislang gegebenen Bedingungskonstellationen als Ausgangspunkt für die weitere Entwicklung, empirisch ausbuchstabiert werden, weil Bedingungslagen pro futuro ermöglichenden und restringierenden Charakter haben. Der Übergang von voraxialen institutionellen Strukturen und Weltdeutungssystemen zu axialen wie auch zu Mischformen kann wohl unterschiedliche Verläufe zeigen, entscheidend ist aber, dass eine neue Gesellschaftsformation mit einem neuen Organisationsprinzip und einem neuen Weltverständnis aufkommt. Wenn Eisenstadt im Anschluss an Jaspers im Falle der Achsenzivilisationen von einer neuen Qualität des Reflexionsvermögens auf die Welt und auf die Gesellschaft spricht, so bleibt doch die Frage des Anschlusses an vorhergehende Gesellschaftsformationen und Weltverständnisse offen bzw. bleibt die prozessuale Rekonstruktion der Dialektik von Kontinuität und Bruch unterbelichtet. Die begrifflichen Unbestimmtheiten, die Kontingenzen in den Prozessen und Strukturen sowie die Fragen des diachronen Anschlusses als auch der synchronen Einbettung der Achsenzivilisationen führten bei Eisenstadt wohl zu Relativierungen und dem Eingeständnis der Notwendigkeit einer differenzierteren Sichtweise (Arnason, Eisenstadt and Wittrock 2005), wurden dann für seine weitere Arbeit aber nicht mehr programmatisch.

Ein weiterer kritischer Einwand zielt darauf ab, dass das Kreativitätsmoment als Zusammenhang von Machtprozessen und deren institutioneller und symbolischer Kristallisation präziser zu fassen ist. Gesellschaftliche Entwicklungen werden wohl über Macht prozessiert, aber in ihren formativen Ausprägungen sind diese auch vom kognitiven Entwicklungsstand abhängig. Während Eisenstadt die den soziokulturellen Prozessen zugrundeliegende Machtdynamik materialreich belegt, bleibt die den axialen Weltbildern zugrundeliegende Denk- und Handlungslogik theoretisch unterbelichtet. Wie aber lässt sich die postulierte neuartige Form der Reflexivität auf die Verhältnisse evolutionstheoretisch rahmen? Diesbezüglich soll nun unter Bezugnahme auf die historisch-genetische Theorie von Günter Dux versucht werden, die logische Struktur der Welterklärungen, so wie sie sich in allen vormodernen Weltbildern, von den pristinen Mythologien bis hin zu den elaborierten Welterklärungen der polytheistischen und monotheistischen Religionen wiederfindet, zu skizzieren. 


\section{Die Entwicklung der kognitiven Matrix differenter Weltbilder}

Die historisch-genetische Theorie von Günter Dux zielt darauf ab, die Bildungsprozesse historisch neuartiger soziokultureller Strukturen und Modelle aus vorwegliegenden Ausgangsbedingungen zu rekonstruieren, die ihrerseits aber die neu entstehenden Merkmale selbst noch nicht aufweisen. Ausgangsbedingungen stellen also Rahmungen dar, innerhalb derer Neuartiges emergieren kann. Die Ausbildung sozialer Organisationsformen wie auch kultureller Orientierungssysteme ist nun unabdingbar eingebettet in den anthropologisch vorgegebenen Zwang, Handlungswissen erwerben zu müssen. Da menschliches Handeln primär kognitiv organisiert und nicht oder kaum instinktgeleitet ist, entscheidet mithin das Niveau der kognitiven Entwicklung, welche Handlungskompetenz zur Verfügung steht und wie die Welt verstanden werden kann.

Empirisch abgesichert ist für Dux, dass die gemeinsame Denkfolie der konstruktiv entstehenden frühen vormodernen Weltinterpretationen strukturell einer subjektivischen Handlungslogik folgt. Erklärungen bedienen sich dabei einer Argumentationsfigur, als ob die zu erklärenden Phänomene von einem handelnden Agens hervorgebracht würden (Dux 1982, S. 76 ff.; 2000, S. 117). Obwohl die subjektivische Handlungslogik in jeder Ontogenese immer wieder von neuem aufgebaut wird, wird damit aber kein Parallelismus von Ontogenese und Geschichte behauptet. Es geht ausschließlich darum, dass sich, ungeachtet der kulturellen Pluralität der Weltdeutungen, auf der Ebene der Weltbilder die Handlungslogik als explikative Struktur bis in die Neuzeit behauptet hat.

Die subjektivische Logik als Denkmatrix ist auch kennzeichnend für die Religionen, deren Aufgabe es ist, die Welt als Ganzes verständlich zu machen. Die kulturgeschichtlich gesehen immer abstrakter werdenden Deutungssysteme bleiben dieser Logik verhaftet. So wird der Ursprung der Welt auf ein subjektives Agens, etwa einen Schöpfergott, zurückgeführt, durch dessen Handeln die Welt entsteht. Damit kann dieser aber zeitlich, kausal und logisch nicht mehr mit der Welt zusammenfallen, er muss dieser vorangestellt werden. Insofern unterscheiden alle Religionen mehr oder weniger elaboriert zwischen zwei Welten, einer dies- und einer jenseitigen Welt. Der Ursprung gewinnt dabei die Qualität eines Absoluten, das aus sich selbst heraus begründet ist (Holz und Wenzel 2003, S. 21 ff.). Geschichtlich wird dieses Weltverständnis immer weiter ausgearbeitet, systematisiert und abstrakt gefasst, was immer komplexere Weltdeutungen erlaubt. Erst in der Neuzeit entsteht mit dem naturwissenschaftlichen Weltbild ein nicht mehr ursprungslogisch ansetzendes Denken, welches vielmehr auf funktional-relationalen Erklärungen aufbaut, in der das Geschehen auf systemisch verstandene Wirkungszusammenhänge zurückgeführt wird (Dux 1982, S. 275 ff.).

Die den Weltbildern immanente Reflexivität kann nicht als ein abstraktes und generalisiertes Vermögen gesetzt werden, sondern ist in ihrer kognitiven Ausprägung soziokulturell rückgebunden. Gesellschaftliche Systeme entwickeln sich aber primär über die dynamische Struk- 
turierung von Interessen und Machtpotentialen. Der sich mit den Jägern- und Sammlerinnengesellschaften in Gang setzende Entwicklungsprozess lässt sich umschreiben als Steigerung der Organisationskompetenz und damit auch des Organisationsniveaus von Gesellschaft. Mit der Sesshaftigkeit entsteht erstmals die Möglichkeit, Macht reflexiv für die Organisation von Machtressourcen, also von Land und von abhängigen Arbeitskräften, zu nutzen. Die Organisation von Macht bringt eine neue Qualität mit sich, sie wird zur Gestaltungsmacht, die in bis dahin unbekannter Art und Weise den Zugriff auf die sozialen Strukturen erlaubt. Bisher latente Abhängigkeiten können auf eine völlig neue institutionelle Basis gestellt werden, es bilden sich stratifizierte Gesellschaften heraus bis hin zu den traditionalen, vormodernen Zivilisationen (Dux 1992).

Irdische Verhältnisse und Erklärungssysteme müssen zur Deckung gebracht werden. Auf der Ebene der Weltbilder hat das tiefgreifende Folgen: die neue Gestaltungsmacht in Bezug auf die soziokulturelle Lebensweise muss reflektiert und ideologisch verarbeitet werden, Herrschaft legitimiert werden. Im Rahmen einer Handlungslogik kann die Legitimation einer Herrschaftsorganisation nicht in abstrakten Prinzipien gründen, sondern muss dem außerweltlichen Ursprung als Quelle jeder Art von Macht verbunden werden. In den nicht mehr verwandtschaftlich, sondern politisch organisierten vormodernen Zivilisationen gibt es dafür Mediatoren, Deutungsspezialisten, die die Anbindung des Mundanen an das Ursprüngliche immer wieder aufs Neue thematisieren. Die den Auslegungen immanente Eigenlogik ist für den herrschaftlichen Bereich nicht mehr uneingeschränkt kontrollierbar. Ein Richtungsstreit zwischen den Experten kann jedoch nur dann als dynamischer Wandlungsfaktor relevant werden, wenn gesellschaftliche Interessengruppen spezifische Deutungen aufgreifen.

Die historisch-genetische Theorie sieht folglich in den praktischen Konsequenzen organisierten Machtgebrauchs das eigentliche Vehikel für die dynamische Weiterentwicklung der Weltbilder, die sich mit neuen normativen Strukturen sowie mit Legitimationsfragen von Herrschaft und Ungleichheit konfrontiert sahen. Die gesteigerte Reflexion auf die Verhältnisse folgt dieser gesellschaftlichen Entwicklungsdynamik, beeinflusst diese aber auch rekursiv.

\section{Folgerungen für eine Theorie langfristigen sozialen Wandels}

Für eine Theorie langfristigen sozialen Wandels im Sinne einer Theorie soziokultureller Evolution erweist sich die Beschäftigung mit der Eisenstadtschen Kultur- und Zivilisationsanalyse in doppelter Hinsicht als fruchtbar: Zum einen sensibilisiert sie dafür, dass es unumgänglich ist, sich mit dem von den historischen Wissenschaften gesammelten Material und deren Interpretationen soziologisch auseinanderzusetzen, um das kategoriale Gerüst der Wandlungs- bzw. Evolutionstheorie sachangemessen zu halten. Angewendet auf die Habermassche Unterscheidung von Gesellschaftsformationen und Organisationsprinzipien mit der dahinterliegenden Lernthe- 
orie bedeutet das, dass dessen Koppelung von Persönlichkeits-, Kultur- und Gesellschaftssystem als evolutionsfähiger Verbund unberücksichtigt lässt, dass die verschiedenen Teilsysteme sich auch in einer gewissen zeitlichen Unabhängigkeit voneinander entwickeln können. Hier ist die Eisenstadtsche Vorstellung von geschichtlichen Tiefendimensionen in Wandlungsprozessen mit ungleichzeitigen Entwicklungen hilfreich, wo moderne und traditionale Elemente in ein und derselben Gesellschaft oder Gruppe Platz finden können (Eisenstadt 1998). Zum anderen macht uns die Eisenstadtsche Zivilisationsanalyse aber auch darauf aufmerksam, dass seine Einordnung und die Bewertung der historischen Bedeutung der Achsenkulturen mit ihren langfristigen Nachwirkungen (Entstehung der Ersten Moderne; These der multiple modernities) erst im Rahmen einer Theorie soziokultureller Evolution wirklich verstanden werden kann. Die von ihm argumentierte Tiefendimension der Moderne in Form der in den Achsenzivilisationen entstandenen Spannungsdimension zwischen Mundanem und Transzendentalem überbetont die Kontinuitätslinie in der Entwicklung hin zur Moderne, während der Bruch im Weltverständnis (Ablösung der subjektivischen durch eine systemische Erklärungslogik) unterbelichtet bleibt.

Was das Weltverständnis betrifft bedarf aber auch die von Eisenstadt als zentrales axiales Merkmal gesetzte Reflexivität auf die Verhältnisse mithin einer evolutionstheoretisch informierten Sichtweise bzw. Erklärung. Nicht eine neue Erklärungslogik zeichnet die gesteigerte Reflexivität in den Achsenzivilisationen aus, sondern die zunehmende Gestaltungsmacht der Menschen, die Trennbarkeit von Natur und Kultur, beginnt ins Bewusstsein zu rücken und bedarf der geistigen Aufarbeitung (vgl. Dux 2000, S. 390 ff.). Die Steigerung der Reflexivität bringt sich in einer Reflexivität auf die Strukturen zum Ausdruck, aber als neuartige Wirklichkeitsauffassung bleibt sie trotzdem handlungslogisch strukturiert. Nach wie vor wird die, Welt als Ganzes' wie ein Objekt behandelt und subjektivisch erklärt (Gott/Götter). Das Moment der Reflexivität auf die Verhältnisse und die Behauptung eines Wissens um die Willkürlichkeit sozialer Ordnung wird bei Eisenstadt (2006, S. 4 ff.) mithin überzeichnet. Dieses trifft nur insofern zu, als dass die konkrete Herrschaftsausübung nunmehr unter Missbrauchsverdacht fallen kann; die Legitimation der gesellschaftlichen Machtverhältnisse als solche bleibt davon jedoch unberührt, das System in seiner Grundarchitektur bleibt unhinterfragt. Gleichzeitig verweist uns dieser Punkt aber auch auf die Ungleichzeitigkeit evolutionärer Prozesse hin, kann man doch nichtsdestotrotz von einer neuen Gesellschaftsformation mit einem neuen Organisationsprinzip sprechen. Dies betrifft auch die legitimatorischen Erfordernisse: Die Legitimation von Herrschaft bedarf letzten Endes des Verweises auf die Ursprungsmacht, aber in der Alltagspraxis hat die gelungene Aufrechterhaltung der sozialen Ordnung ebenso einen legitimationsstiftenden Charakter, etwas, was Popitz (1992) als Ordnungssicherheit bezeichnet hat und womit sich die innere Stabilität von Macht- und Herrschaftssystemen auch unabhängig von ideologischen Stützkonstruktionen erklären lässt.

Welche Bedeutung haben dann aber kulturelle Orientierungen, Weltbilder für den Prozess des sozialen Wandels? Sie können die Grundlagen konkreter Herrschaftspraxen unterminieren, 
indem sie ihnen den legitimatorischen Bezug zum Ursprung allen Seins entziehen; der spezialistische Streit um das richtige Verständnis kann von machtpolitisch interessierten Gruppen aufgegriffen werden, um eigenen Interessen eine legitimatorische Basis zu geben; andere Konstellationen lassen sich denken. Für das Verhältnis von Macht und Kultur bedeutet das immer nur eines: Im Rahmen der Denklogik geht es nicht um ein neues System, sondern es geht um die Adaptation des vorhandenen an kulturelle Basisparameter, wie sie die Weltbilder liefern. Konkret heißt das also, Austausch von Personen oder Herrschaftseliten, die dem Maßstab nicht entsprechen. Einige Unterscheidungen scheinen in dieser Hinsicht angebracht: In systeminternen Konflikten werden kulturelle Deutungen eher instrumentellen Charakter für die jeweiligen Konfliktgruppen haben, in extern induzierten Bedrohungslagen, die Eisenstadt weitgehend ausspart, haben kulturelle Orientierungen eher katalysatorische Funktionen für die Organisation des Widerstandes, indem sie die kollektive Identität und damit gleich gelagerte Interessen beschwören, und in der Expansion nach Außen haben sie vermutlich sowohl instrumentelle als auch katalysatorische Funktionen. Für die von Eisenstadt angenommene Verschmelzung von Macht und Kultur als der wesentlichen Triebkraft sozialen Wandels heißt das, dass in konflikttheoretischer Perspektive erst in gesellschaftlichen Krisensituationen der Kultur eine zentrale Rolle zugemessen werden kann. In der Realität gesellschaftlichen Lebens sind Fragen der Machtverteilung, die Infragestellung herrschaftlicher Ansprüche und die Unzufriedenheit mit den Folgen der empirisch vorgefunden Machtverwaltung wohl vorrangigere Erklärungsfaktoren als die isoliert betrachtete Spannung zwischen Realität und Transzendenz.

Mit Skepsis ist schließlich auch der Eisenstadtschen Vorstellung zu begegnen, dass sich aus der variantenreichen Auflösung der strukturellen Spannung zwischen Realität und Transzendenz in den Achsenzivilisationen multiple Modernen begründen lassen. Hier wird das Spannungsverhältnis zwischen transzendentaler Vision und innerweltlichen Institutionalisierung, wie es in den Achsenzivilisationen aufgetreten ist, als Wandlungsmechanismus invariant gesetzt (Bohmann 2003, S. 242). Selbst, wenn auf der analytischen Ebene des Strukturvergleichs die-

ses Spannungsverhältnis als Tiefendimension nachweisbar ist: Prozesse langfristigen sozialen Wandels folgen gemeinhin einer anderen Logik, und die ist nur evolutionstheoretisch einholbar.

\section{Literatur}

Arnason, Johann P., Shmuel N. Eisenstadt and Björn Wittrock (eds.). 2005. Axial Civilizations and World History. Leiden, Boston: Brill.

Bohmann, Gerda. 2003. Fundamentalismus als Stadium sozialer Evolution? In: Soziale Evolution, hrsg. Tamás Meleghy und Heinz-Jürgen Niedenzu, 239-266. Wiesbaden: Westdeutscher Verlag.

Dux, Günter. 1982. Die Logik der Weltbilder. Sinnstrukturen im Wandel der Geschichte. Frankfurt am Main: Suhrkamp. 
Dux, Günter. 1992. Die Spur der Macht im Verhältnis der Geschlechter. Frankfurt am Main: Suhrkamp.

Dux, Günter. 1989. Die Zeit in der Geschichte. Ihre Entwicklungslogik vom Mythos zur Weltzeit. Frankfurt am Main: Suhrkamp.

Dux, Günter. 2000. Historisch-genetische Theorie der Kultur. Instabile Welten - Zur prozessualen Logik im kulturellen Wandel. Weilerswist: Velbrück.

Eder, Klaus. 1973. Seminar: Die Entstehung von Klassengesellschaften. Frankfurt am Main: Suhrkamp.

Eder, Klaus. 1976. Die Entstehung staatlich organisierter Gesellschaften. Frankfurt am Main: Suhrkamp.

Eisenstadt, Shmuel N. 1970. Sozialer Wandel, Differenzierung und Evolution. In: Theorien des sozialen Wandels, hrsg. Wolfgang Zapf, 75-91. Köln/Berlin: Kiepenheuer \& Witsch.

Eisenstadt, Shmuel N. 1998. Die Antinomien der Moderne. Die jakobinischen Grundzüge der Moderne und des Fundamentalismus. Heterodoxien, Utopismus und Jakobinimus in der Konstitution fundamentalistischer Bewegungen. Frankfurt am Main: Suhrkamp.

Eisenstadt, Shmuel N. 2006. Culture and Power - A Comparative Civilizational Analysis. Erwägen - Wissen-Ethik 17: 3-16.

Eisenstadt, Shmuel N. 2009. Axial Visions and Axial Civilizations: The Transformations of World Histories between Evolutionary Tendencies and Institutional Formations. In: Frontiers of Sociology. Annals of the International Institute of Sociology, Vol.11, hrsg. Peter Hedström und Björn Wittrock, 113-146. Leiden: Brill.

Elias, Norbert. 1983. Gedanken über die große Evolution. Zwei Fragmente. In: Engagement und Distanzierung. Arbeiten zur Wissenssoziologie I, ders., 185-268. Frankfurt am Main: Suhrkamp.

Elias, Norbert. ${ }^{2}$ 1989. Über die Zeit. Frankfurt am Main: Suhrkamp.

Habermas, Jürgen. ${ }^{3} 1982$ [1976]. Zur Rekonstruktion des Historischen Materialismus. Frankfurt am Main: Suhrkamp.

Habermas, Jürgen. ${ }^{3} 1982$ a [1976]. Geschichte und Evolution. In: Zur Rekonstruktion des Historischen Materialismus. ders., 200-259. Frankfurt am Main: Suhrkamp.

Habermas, Jürgen. ${ }^{3} 1982$ b [1976]. Zum Theorienvergleich in der Soziologie: am Beispiel der Evolutionstheorie. In: Zur Rekonstruktion des Historischen Materialismus, ders., 129-143. Frankfurt am Main: Suhrkamp.

Holz, Klaus und Ulrich Wenzel. 2003. Einleitung. Handlungen und Subjekte in der historisch-genetischen Theorie. In: Zur Konstitution von Sozialität, hrsg. Ulrich Wenzel et al., 9-42. Velbrück: Weilerswist.

Meleghy, Tamás und Heinz-Jürgen Niedenzu (Hrsg.). 2003. Soziale Evolution. Die Evolutionstheorie und die Sozialwissenschaften (ÖZS-Sonderband 7). Wiesbaden: Westdeutscher Verlag.

Niedenzu, Heinz-Jürgen. 2012. Soziogenese der Normativität. Zur Emergenz eines neuen Modus der Sozialorganisation. Weilerswist: Velbrück.

Niedenzu, Heinz-Jürgen. 2014. Ursprung und Wandel der Gesellschaft. Zum soziologischen Verständnis geschichtlicher Prozesse. In: Entwicklungen der Menschheit. Humanwissenschaften in der Perspektive der Integration, hrsg. Gerd Jüttemann, 111-118. Lengerich: Pabst Science Publishers. 
Niedenzu, Heinz-Jürgen. ${ }^{112016}$. Evolution, soziale. In: Grundbegriffe der Soziologie, Johannes Kopp und Anja Steinbach, 73-76. Wiesbaden: Springer VS.

Parsons, Talcott. 1977. The Evolution of Societies. Englewood Cliffs, NJ: Prentice-Hall.

Popitz, Heinrich. ${ }^{2}$ 1992. Phänomene der Macht. Tübingen: Mohr.

Preglau, Max. ${ }^{9}$ 2015. Kritische Theorie: Jürgen Habermas. In: Soziologische Theorie. Abriss der Ansätze ihrer Hauptvertreter, hrsg. Julius Morel et al., 301-330. Berlin/Boston: Walter de Gruyter.

Reichholf, Josef H. 1994. Der schöpferische Impuls. München: dtv.

Runciman, Walter G. 1989. A Treatise on Social Theory. Vol II: Substantive Social Theory. Cambridge University Press.

Runciman, Walter G. 2009. The Theory of Cultural and Social Selection. Cambridge University Press.

Sanderson, St. K. 1990. Social Evolutionism. A Critical History. Cambridge, MA; Oxford, UK: Blackwell Publishers.

Sanderson, Stephen K. 2001. The Evolution of Human Sociality. A Darwinian Conflict Perspective. Lanham et al.: Rowman \& Littlefield Publishers.

Sanderson, Stephen K. and Arthur S. Alderson. 2005. World Societies. The Evolution of Human Social Life. Boston et al.: Pearson Education.

Heinz-Jürgen Niedenzu, ist außerordentlicher Universitätsprofessor am Institut für Soziologie der Universität Innsbruck. Arbeitsgebiete/Forschungsschwerpunkte: Soziologische Theorie, mit Schwerpunkt auf Fragen nach den anthropologischen Grundlagen der Sozialtheorie, der Entwicklung einer Theorie soziokultureller Evolution sowie von Modellen des langfristigen Wandels gesellschaftlicher Strukturen. Ausgewählte Publikationen: The new evolutionary social science: human nature, social behaviour and social change. Boulder/London: Paradigm Publishers, 2008 (hrsg. mit Meleghy, T. und Meyer, P.); Soziogenese der Normativität. Zur Emergenz eines neuen Modus der Sozialorganisation. Weilerswist: Velbrück, 2012; Ursprung und Wandel der Gesellschaft. Zum soziologischen Verständnis geschichtlicher Prozesse, in: Jüttemann, Gerd (Hrsg.): Entwicklungen der Menschheit. Humanwissenschaften in der Perspektive der Integration. Lengerich: Pabst Science Publishers, 2014, S. 111-118; Evolution, soziale, in: Kopp, Johannes / Steinbach, Anja (Hrsg.): Grundbegriffe der Soziologie, 11.Auflage. Wiesbaden: Springer VS 2016, S. 73-76. 